\title{
On the temperature and stress dependence of transformation strain in single crystalline $\mathrm{Cu}-\mathrm{Al}-\mathrm{Ni}$ shape memory alloys
}

\author{
D.L. Beke*, T.Y. El Rasasi, L. Daróczi, \\ Department of Solid State Physics, University of Debrecen, Hungary H-4010, Debrecen,
}

\begin{abstract}
In the austenite to martensite phase transformation of shape memory alloys the transformation strain, $\varepsilon^{t r}$, usually has definite dependence on the external fields while the change in entropy or volume can be considered constant. Under uniaxial loading this dependence of $\varepsilon^{t r}$ is related to the change of the martensite variant distribution with increasing field parameters. The stress and temperature dependence of the elastic and dissipative energy contributions to the transformation can be correlated with the $\eta$ dependence of $\varepsilon^{d r}$, where $\eta$ is the volume fraction of the stress induced (single) variant martensite structure. Obviously, this dependence leads to deviation from the linear Clausius-Clapeyron relation too. In this paper we discuss and compare experimental results in single crystalline $\mathrm{Cu}-$ $17.9 \mathrm{wt} . \% \mathrm{Al}-2.6 \mathrm{wt} . \% \mathrm{Ni}$ and $\mathrm{Cu}-11.5 \mathrm{wt} \%-\mathrm{Al} 5.0 \mathrm{wt} \% \mathrm{Ni}$ alloys obtained from stress-strain and strain-temperature hysteretic loops at different constant temperatures and stresses, respectively. Furthermore, it is shown that the field dependence of $\varepsilon^{t r}$ is reflected in the field dependence of the dissipative and elastic energy terms too.
\end{abstract}

\section{Introduction}

The problem of stress, $\sigma$, and temperature, $T$, dependence of the transformation strain, $\varepsilon^{t r}$, in martensitic transformations of shape memory alloys is considered. In many papers on the subject (see e.g. [1]) it is assumed that the transformation strain is constant. On the other hand there are publications [2-5] in which the dependence of $\varepsilon^{t r}$ on the field variables, $T$ and $\sigma$, is considered. In our recently published paper [6] we included the volume fraction of the stress induced (single) variant martensite structure, $\eta$, into our model [7,8,9], which offers a simple way for the separation of the elastic and dissipative contributions to the start and finish temperatures and stresses. Here $\eta=V_{M \sigma} / V_{M}, V_{M}=V_{M T}+V_{M \sigma}$ and $\xi=V_{M} / V$ is the martensite volume fraction, with $V=V_{M}+V_{A}\left(V_{M}\right.$ and $V_{A}$ are the volume of the martensite and austenite phases, respectively and $V_{M T}$ and $V_{M \sigma}$ denotes the volume of the thermally as well as the stress induced martensite variants, respectively). Obviously $\xi=\xi_{T}+\xi_{\sigma}=\xi(1-\eta)+\xi \eta$, where e.g. $\xi_{\sigma}=V_{M \sigma} / V$. The analysis of different hysteretic curves can bring important contributions about the role of chemical, dissipative and elastic terms to the transformation and can lead to improved control of the shape memory function $[8,10]$.

The organization of the paper is as follows. In Sec. 2 the main concept of our model is summarized and relations used in the analysis of experimental data are given. In Sec.3 our experimental data on the $\sigma-\varepsilon$ and $\varepsilon-T$ hysteretic curves in single crystalline $\mathrm{Cu}-17.9 \mathrm{wt} . \% \mathrm{Al}-2.6 \mathrm{wt} . \% \mathrm{Ni}$ [7] and $\mathrm{Cu}-11.5 \mathrm{wt} \%-\mathrm{Al} 5.0 \mathrm{wt} \% \mathrm{Ni}$ alloys [11] are analyzed and compared, giving a special emphasis to the consequence of the field dependence of $\varepsilon^{t r}$. Sec. 4 and 5 contain comparison with other data from the literature and the conclusions, respectively.

\section{Relations used in the analysis of data}

The model used is in fact a local equilibrium formalism and based on the thermoelastic balance [1, 12] and starts from the following relation (see also $[6,8]$ ) for the change of the Gibbs free energy per mole

$$
\partial\left(\Delta G^{\downarrow}\right) / \partial \xi=\Delta g^{\downarrow}+e^{\downarrow}(\xi)+d^{\downarrow}(\xi)=0,
$$

where $\Delta g, e$ and $d$ are the derivatives of the changes in chemical free energy, $\Delta G_{\mathrm{ch}}$, elastic, $E$, and dissipative energy, $D$, by $\xi$. The down arrow indicates that (1) is written for the austenite to martensite transition and a

*email: dbeke@delfin.unideb.hu

This is an Open Access article distributed under the terms of the Creative Commons Attribution-Noncommercial License (http://creativecommons.org/licenses/by-nc/3.0/), which permits unrestricted use, distribution, and reproduction in any noncommercial medium, provided the original work is properly cited. 
similar relation holds for the reverse process with arrow up. $\Delta g$ can have the general form as (for the sake of simplicity, the up and down arrow is omitted):

$$
\Delta g=\Delta u-T \Delta s-\sigma V \varepsilon^{t r}+p \Delta V-B \Delta M
$$

where $\Delta s=\Delta s^{\downarrow}\left(=s_{M}-s_{A}=-\Delta s^{\uparrow}(<0)\right)$ is the entropy change per mole, $\varepsilon^{\text {tr }}$ is the transformation strain, $V$ is the molar volume, as well as $\Delta V$ and $\Delta M$ denote the change in the volume and magnetization, respectively. $p$ and $B$ have their usual meaning. In the following only the effects of uniaxial stress and temperature will be considered, thus only the first two terms will be kept.

In [6] only the field dependence of $\varepsilon^{t r}$ was treated and $\Delta u$ and $\Delta s$ were taken to be constant. In principle the term containing the stress has tensor character and, as a consequence, even if one considers uniaxial loading conditions (leading to scalar term in (2)) the field dependence of $\varepsilon^{\text {tr }}$ is related to the change of the martensite variant distribution with increasing field parameters. Thus at zero $\sigma$ values thermally oriented multi-variant martensite structure forms, while at high enough values of $\sigma$ a well oriented array i.e. a single variant structure develops. It was already explicitly assumed in [11] that the field dependence of $\varepsilon^{\text {tr }}$ is the consequence of its dependence on $\eta$, and thus here for the sake of clarity and simplicity in all relations $\varepsilon^{\text {tr }}$ will be considered always at that $\eta$ which belongs the corresponding fixed values of the field parameters.

The definitions of the equilibrium transformation temperature, $T_{o}$, and stress, $\sigma_{o}$, give

and thus

$$
\begin{aligned}
& \Delta g=\Delta u-T_{o} \Delta s=0 \quad(\text { at } \sigma=0), \quad T_{o}(0)=\Delta u / \Delta s, \\
& \Delta \mathrm{g}=\Delta \mathrm{u}-\sigma \mathrm{V} \varepsilon^{\mathrm{tr}} . \quad(\text { at } \mathrm{T}=0), \quad \sigma_{\mathrm{o}}(0)=\Delta \mathrm{u} / V \varepsilon^{t r}
\end{aligned}
$$

$$
\sigma_{o}(0)=T_{o}(0) \Delta s / V \varepsilon^{t r}
$$

In these relations $\varepsilon^{t r}$ should correspond to that value which corresponds to the martensite variant structure (and thus to a given $\eta$ value) formed at zero temperature during stress induced phase transformation.

It was shown in [1], that the condition (1), using relations (3)-(5), gives the following expressions

$$
\begin{aligned}
& T^{\downarrow}(\xi)=T_{o}(\sigma)-\left[d^{\downarrow}(\xi)+e^{\downarrow}(\xi)\right] /[-\Delta s] \\
& T^{\uparrow}(\xi)=T_{o}(\sigma)+\left[d^{\uparrow}(\xi)+e^{\uparrow}(\xi)\right] /[-\Delta s],
\end{aligned}
$$

where again the up and down arrows correspond to the cooling and heating process, respectively. In fact the inverses of these functions (i.e. the $\xi\left(T^{\downarrow}\right)$ and $\xi\left(T^{\uparrow}\right)$ curves) are the lower and upper parts of the normalized $\varepsilon$-T hysteretic loops, respectively. Thus, making the usual assumptions $e(\xi)=e(\xi)^{\downarrow}=-e(\xi)^{\uparrow}$ and $d(\xi)=d(\xi)^{\downarrow}=d(\xi)^{\uparrow}$ and taking the difference and the sum of $T^{\downarrow}(\xi)$ and $T^{\uparrow}(\xi)$, the $e$ and $d$ quantities can be calculated as the function of $\xi$ at different $\sigma$ levels. (Note that the $e(\xi)$ function can be calculated irrespective of the $T_{o}(\sigma) \Delta s$ constant value $[9,11])$, if $T_{\mathrm{o}}(0)$ is not known.). Similar relations can be written for the $\sigma-\varepsilon$ hysteretic loops [6]. In $(6) T_{o}(\sigma)$ is the stress dependent equilibrium transformation temperature and is given by

$$
T_{o}(\sigma)=T_{o}(0)-(1 / \Delta s) \sigma V \varepsilon^{t r}
$$

which is the integral form of the of the Clausius-Clapeyron relation. Here $\varepsilon^{\text {tr }}$ should depend on the stress (bay means of $\eta$ ) at which the given martensite variant structure develops in the temperature induced phase transformation. From the analogous relation for the $\sigma-\varepsilon$ hysteretic loops

$$
\sigma_{o}(T)=-\left(\Delta s / V \varepsilon^{t r}\right)\left[T-T_{o}(0)\right]
$$

with temperature dependent $\varepsilon^{t r}$ belonging to the martensite variant structure (and the corresponding $\eta$ ) which develops during the stress induced phase transformation at a given temperature.

Now taking the relations (6) (and the analogous ones for the $\sigma-\varepsilon$ hysteretic loops) at the start and finish points (i.e. at $\xi=0$, and $\xi=1$ ) one arrives at the following relations for the martensite and austenite start and finish temperatures $\left(M_{s}, M_{f}, A_{s}, A_{f}\right)$ and stresses $\left(\sigma_{M s}, \sigma_{M f}, \sigma_{A s}, \sigma_{A f}\right)$ :

$$
\begin{aligned}
& M_{s}(\sigma)=T_{o}(\sigma)-\left[d_{o}+e_{o}\right] /[-\Delta s] \\
& M_{f}(\sigma)=T_{o}(\sigma)-\left[d_{1}+e_{1}\right] /[-\Delta s]
\end{aligned}
$$


as well as

$$
\begin{aligned}
& A_{f}(\sigma)=T_{o}(\sigma)+\left[d_{o}-e_{o}\right] /[-\Delta s] \\
& A_{s}(\sigma)=T_{o}(\sigma)+\left[d_{l}-e_{I}\right] /[-\Delta s]
\end{aligned}
$$

$$
\begin{aligned}
& \sigma_{M s}(T)=\sigma_{o}(T)-\left[d_{o}+e_{o}\right] /\left[-V \varepsilon^{t r}\right] \\
& \sigma_{M f}(T)=\sigma_{o}(T)-\left[d_{1}+e_{1}\right] /\left[-V \varepsilon^{t r}\right] \\
& \sigma_{A f}(T)=\sigma_{o}(T)+\left[d_{o}-e_{o}\right] /\left[-V \varepsilon^{t r}\right] \\
& \sigma_{A s}(T)=\sigma_{o}(T)+\left[d_{1}-e_{l}\right] /\left[-V \varepsilon^{t r}\right] .
\end{aligned}
$$

Finally it was proposed in [6] that the stress and/or temperature dependence of $\varepsilon^{t r}$ can be expressed by means of the $\eta$ dependence as:

$$
\varepsilon^{t r}=\varepsilon_{T}+\left(\varepsilon_{\sigma}-\varepsilon_{T}\right) \eta
$$

where $\varepsilon_{T}$ and $\varepsilon_{\sigma}$ are the transformation strains when fully thermally induced multi variant structure forms $(\eta=0)$, as well as when the martensite consists of a fully ordered array of stress preferred variants (single variant state, $\eta=1$ ), respectively. As it was already treated above, this is plausible because usually $\eta$ (and thus $\varepsilon^{t r}$ ) is not constant [6] but can depend on the $\mathrm{T}$ and $\sigma$ variables. It can be very small or even close to zero for the formation of the thermally induced (randomly oriented) martensite variants (usually there is a very small resultant (remanent) strain in single crystalline samples). On the other hand during the formation of stress induced martensite a single variant structure can form $(\eta=1)$ i.e. $\varepsilon^{t r}=\varepsilon_{\sigma}$.

The stress or temperature dependence of $\eta$ leads, according to (11), to deviations from the linear behaviour in Clausius-Clapeyron relations ((7) and (8)). It was also proposed in [6] that the field dependence of the dissipative and elastic energy contributions (the $d_{i}$ and $e_{i}$ quantities) can also be related to the given martensite variant structure (i.e. to $\eta$ ) which develops during the phase transformation and the $\eta$ dependence of $d_{i}$ and $e_{i}$ can be described by similar relations like (11). These questions will be analyzed in this article by the examples of two types of martensitic phase transformation in $\mathrm{Cu}-\mathrm{Al}-\mathrm{Ni}$ single crystalline alloys undergoing transformation from the high temperature cubic $\beta$ phase to the $\beta^{\prime}(18 \mathrm{R})$ phase [11] as well as to the orthorhombic $\chi(2 \mathrm{H})$ phase [6] (hereafter they will be referred as $\beta / \beta$ ' as well as $\beta / \gamma$ phase transformations), respectively .

\section{Analysis of the experimental data}

Let us first investigate the stress and temperature dependence of the transformation strain. In [6] it was obtained that it had S-shape dependence on the stress (Fig. 1) for the $\beta / \gamma$ phase transformation in a single crystal with orientation $[110]_{\mathrm{A}}$. Thermal hysteretic loops were also investigated and it was shown that, due to the special shapes of the above loops (having rectangular parts), the determination of the equilibrium transformation temperature, $T_{o}$, and its the stress dependence was also possible (Fig. 2). It can be seen that the points obtained directly form the vertical parts of the normalized hysteretic loops (in these parts there is no elastic energy contribution and the application of relations like the one proposed by Tong and Wayman [13] $\left(T_{o}=\left[M_{S}+A_{f}\right] / 2\right)$ could be applied; see also [14]) and the points calculated from (7) using the measured entropy change and the data shown in Fig. 1 fit very well to each other. It is clear that this is not a linear function.

On the other hand Fig. 3 shows the stress dependence of the transformation strain for the $\beta / \beta$, transformation [11]. It can be seen that it has a similar shape as the curve in Fig.1. It is worth mentioning that on both Fig. 1 and 3 the saturation value, $\varepsilon_{\text {sat }}$, is approximately equal to the maximal possible transformation strain corresponding to the estimated value for the case when a single crystal fully transforms to the most preferably oriented martensite. Accordingly we can assume that $\varepsilon_{s a t}=\varepsilon_{\sigma}=\varepsilon_{\text {max }}^{t r}$

Fig. 4 shows the stress dependence of $T_{o}$ for the $\beta / \beta$ ' transformation. In this case the determination of absolute values was not possible, but the $T_{o}(\sigma)-T_{o}(0)$ difference could be calculated using the measured $\Delta \mathrm{s}$ value and the $\varepsilon^{t r}(\sigma)$ curve. It can be seen that this function can be approximated by a straight line in the entire stress interval. But, as it is illustrated in the insert of this figure, if we plot this function only at low stresses than an S-shape dependence appears. Thus it can be concluded, in contrast to the very frequently used approximation in the literature $[1,15,16]$ about linear Calusius-Clapeyron relations, that the $\sigma$ dependent $\varepsilon^{t r}$ usually leads to nonlinear dependence. Of course in special cases, i.e. when the dependence of $\varepsilon^{t r}$ in the investigated range is week, or the stress interval wide enough to have many points belonging to the saturation value of $\varepsilon^{t r}$ a linear fit with an effective slope can be made, like in Fig. 4. The slope of this straight line is $0.90 \mathrm{~K} / \mathrm{MPa}$, which corresponds to an effective constant $\varepsilon^{\text {tr }}$ value in equation (7) equal to $0.065\left(\Delta s=-7.2 \times 10^{4} \mathrm{~J} / \mathrm{Km}^{3}[11]\right)$, which is a bit larger that $\varepsilon^{t r}$ sat $=0.061[11]$. 
Fig. 5 shows the temperature dependence of $\varepsilon^{t r}$ for the $\beta / \beta^{\prime}$ transformation as determined from the measured $\sigma-\varepsilon$ loops [11]. It can be seen that, although it was mentioned in [6] that during stress induced phase transformations the temperature dependence of $\varepsilon^{t r}$ can be very week and its value can be close to $\varepsilon^{t r}{ }_{\text {sat }}$, the transformation strain has a considerable temperature dependence. As a consequence, according to equation (8) the equilibrium transformation stress should be non-linear function of temperature. Unfortunately, here it was not possible to use a similar estimation which has been done for the stress dependence of $T_{o}(0)$ in [6], because the value of $T_{o}(0)$ was not known (see also eq. (8)).

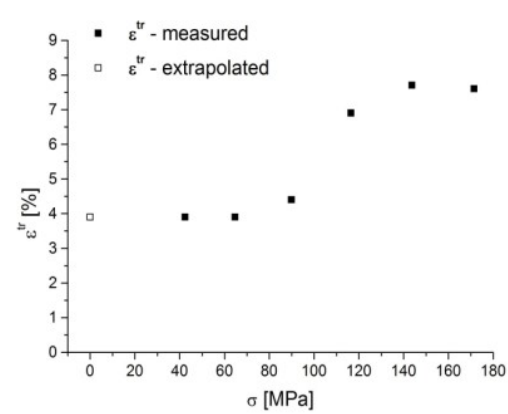

Fig. 1. $\varepsilon^{\text {tr }}(\sigma)$ function in single crystalline $\mathrm{Cu}-$ $17.9 \mathrm{wt} \% \mathrm{Al}-2.6 \mathrm{wt} \% \mathrm{Ni}$ alloy (for $\beta / \gamma$ phase transformation)

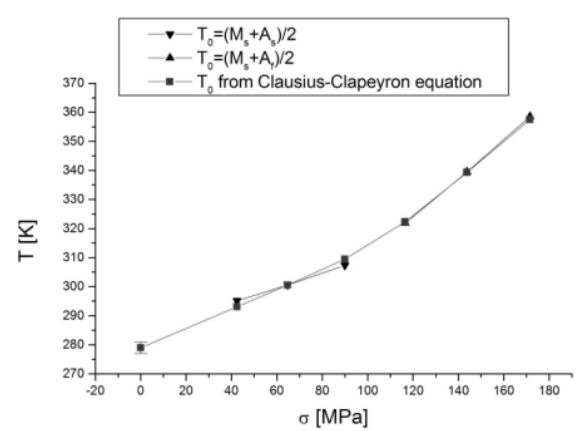

Fig. 2. Stress dependence the equilibrium transformation temperature, $T_{o}$, in single crystalline $\mathrm{Cu}-17.9 \mathrm{wt} \% \mathrm{Al}-2.6 \mathrm{wt} \% \mathrm{Ni}$ alloy (for $\beta / \gamma$ phase transformation; see also the text)

It is clear that $\eta$ can be calculated as the function of $\sigma$ and $T$ from Figs. 1, 3 and 5, respectively, using relation (11). This indicates that a fully ordered single variant martensite structure develops above $140 \mathrm{MPa}$ for the $\beta / \gamma$ phase transformation, while for the $\beta / \beta$ ' transformation $\eta$ is about $80 \%$ already for $28 \mathrm{MPa}$ and then gradually increases up to $100 \%$ in the $40-178 \mathrm{MPa}$ interval. As regards the temperature dependence of $\eta$, it can be seen from Fig. 5 that (according to eq. (11) $\varepsilon^{t r}{ }_{T} \cong 0$, and $\left.\varepsilon^{t r}{ }_{\sigma}=0.061\right) \eta$ monotonously increases from about $10 \%$ up to $100 \%$ between 350 and $430 \mathrm{~K}$.

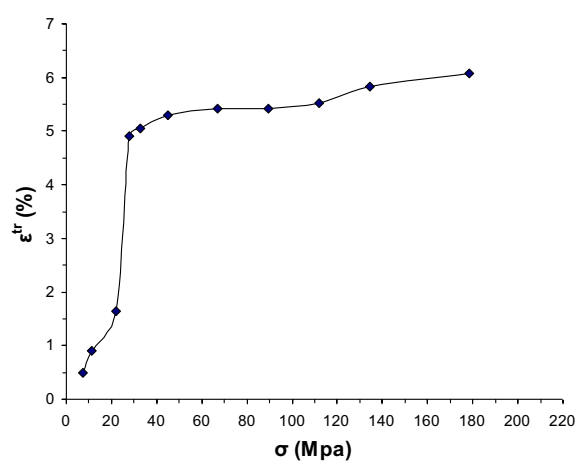

Fig. 3. $\varepsilon^{t r}(\sigma)$ function in single crystalline $\mathrm{Cu}-$ $11.5 \mathrm{wt} \%-\mathrm{Al} 5.0 \mathrm{wt} \% \mathrm{Ni}$ alloy (for $\beta / \beta$ ' phase transformation)

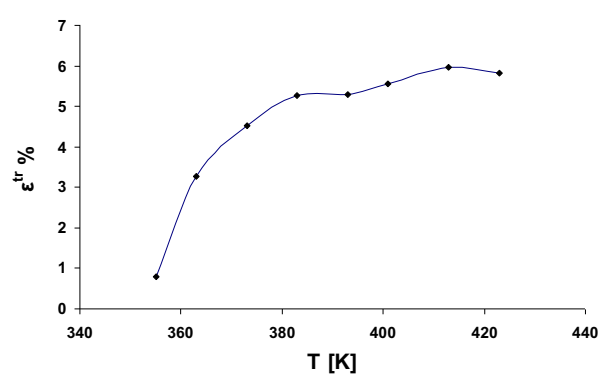

Figure 5. $\varepsilon^{t r}(T)$ function in single crystalline $\mathrm{Cu}-$ $11.5 \mathrm{wt} \%$-Al5.0wt\%Ni alloy (for $\beta / \beta$ 'phase

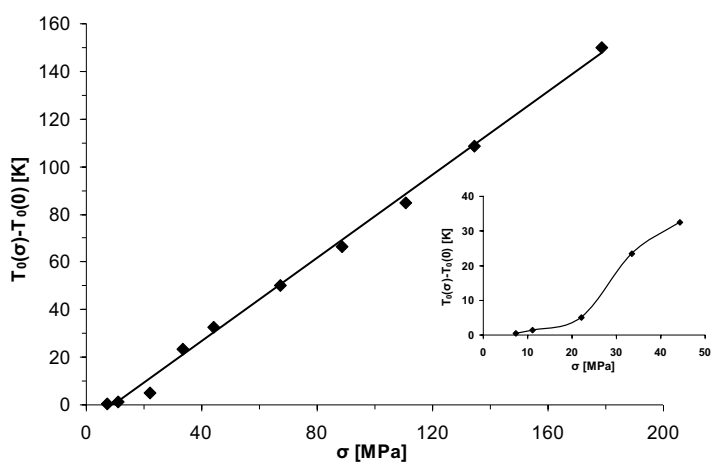

Fig. 4. Stress dependence the equilibrium transformation temperature, $T_{o}$, in single crystalline $\mathrm{Cu}-11.5 \mathrm{wt} \%-\mathrm{Al} 5.0 \mathrm{wt} \% \mathrm{Ni}$ alloy (for $\beta / \beta$ ' phase transformation; see also the text)

It is also worth investigating whether the field dependence of $\eta$ is reflected in the stress and temperature dependence of the dissipative and elastic energy terms or not. Figure $6 \mathrm{a}$ and $6 \mathrm{~b}$ show the stress dependence of the dissipative term, $d=d_{o} \simeq d_{1}$ as well the integral value of the dissipative and elastic energy (for one thermal cycle) $D$ and $E$, respectively for the $\beta / \gamma$ phase transformation [7]. These curves should be compared with Fig. 1. It can be seen that the non-linear shapes of these functions are correlated with the $\varepsilon^{t r}(\sigma)$ curve since the main changes lie in the same stress interval in all cases. 

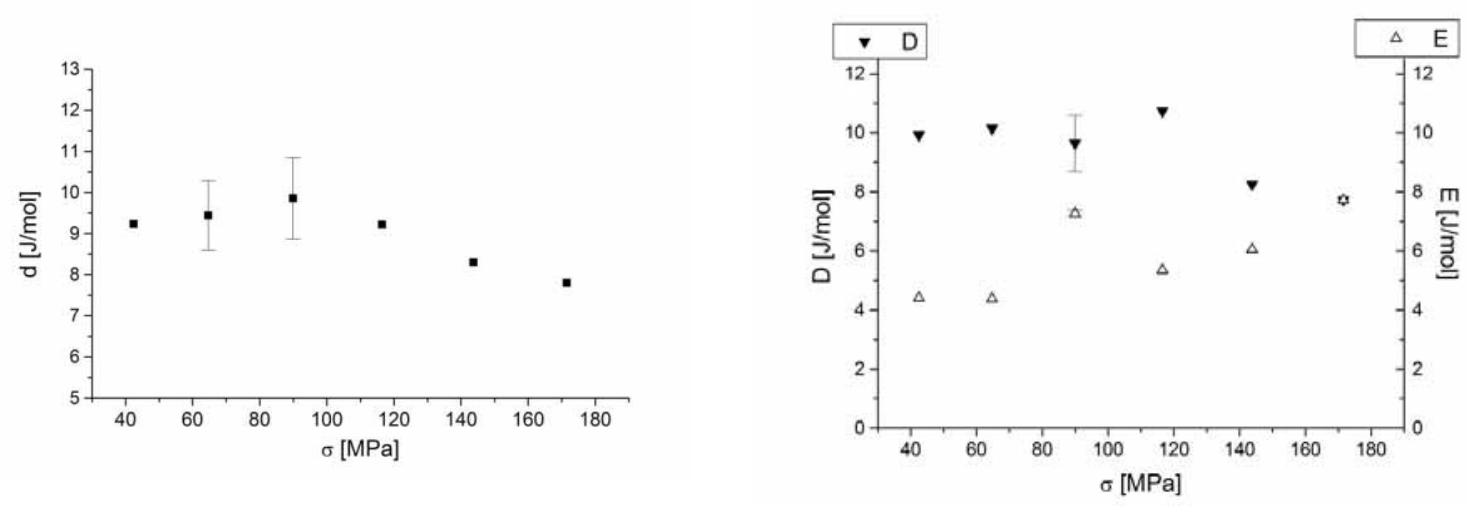

Fig. 6. a) Stress dependence of the derivative of the dissipative energy $\left(d=d_{o} \simeq d_{1}\right)$; b) stress dependence of the integral of the dissipative and elastic energies $D$ and $E$, for one cycle in $\beta / \gamma$ phase transformation.

A similar comparison can be made for the dissipative energy contributions for the $\beta / \beta$ ' transformation. Fig. 7, which shows the $d_{o}$ and $d_{l}$ as well as $D$ quantities versus $\sigma$ should be compared with Fig. 3 . It can be seen that, although the scatter in the experimental points is high enough making difficult to get such an equivocal conclusion as above, there is again a correlation (the changes are mostly below $40 \mathrm{MPa}$ as it is also shown in the insert of Fig. 7b).
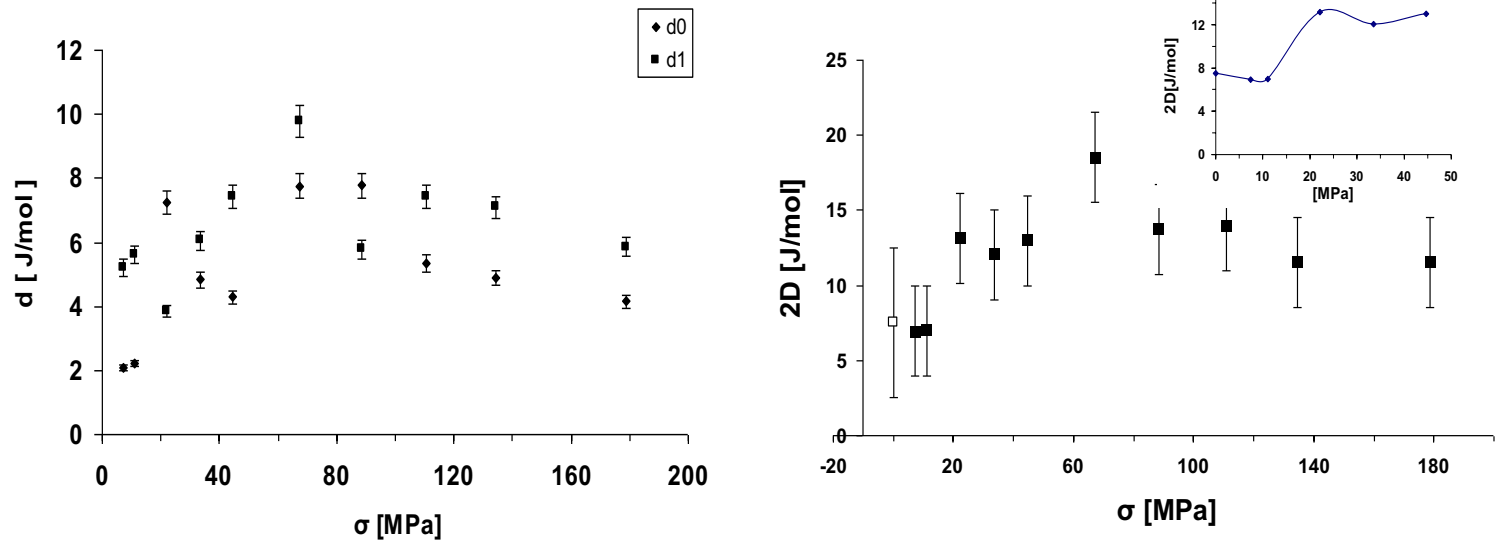

Fig. 7 Stress dependence of the derivative of the dissipative energy $\left(d=d_{o} \simeq d_{1}\right)$; b) stress dependence of the integral of the dissipative energy $D$, for one cycle in $\beta / \beta$ ' phase transformation.

Finally, it is worth mentioning that, as it can be seen from the relations (7) and (9) as well as from (8) and (10), that the stress dependence of the start and finish temperatures or the temperature dependence of the start and finish stresses in general should be different from the stress dependence of $T_{o}$ as well as the temperature dependence of $\sigma_{o}$. Thus even if these functions are approximately linear, their slopes are different. This was nicely illustrated in [11] and even it was shown that this difference was attributed to the stress dependence of the elastic terms. As a typical questionable approach, related to the above problem, is for example the application of the relation

$$
\sigma_{M s}(T)=-\left(\Delta s / V \varepsilon^{t r}\right)\left[T-M_{s}(0)\right]
$$

for the determination of the entropy change $[12,17]$. Thus the observation that for different crystal orientation the slope is different [3], do not mean different values of $\Delta s$, but rather related to different $\varepsilon^{t r}\left(\sigma_{M s}\right)$, because this can be different for different orientations. 


\section{Conclusions}

It is shown that in single crystalline $\mathrm{Cu}-17.9 \mathrm{wt} \% \mathrm{Al}-2.6 \mathrm{wt} \% \mathrm{Ni}$ and $\mathrm{Cu}-11.5 \mathrm{wt} \%-\mathrm{Al} 5.0 \mathrm{wt} \% \mathrm{Ni}$ alloys, during the $\beta / \gamma$ and $\beta / \beta$ ' phase transformations, respectively, the transformation strain, $\varepsilon^{t r}$, has a definite stress and temperature dependence. This is interpreted as the change of the volume fraction of the stress induced martensite variant structure, $\eta$; e.g. at low stress level (in taking the $\varepsilon-T$ loops) dominantly thermally induced multi-variant martensite structure forms, while at high enough values of $\sigma$ a well oriented array i.e. a single variant (or single domain structure) develops. Thus we assumed that $\varepsilon^{t r}$ is the function of $\eta$ and the $\sigma$ and $T$ dependence of $\varepsilon^{t r}$ can be described by means of $\eta(\sigma, T)$.

The field dependence of $\varepsilon^{t r}$ leads to deviations form the linear behaviour in the Clausius-Clapeyron relation. Furthermore, it is reflected in the field dependence of the dissipative and elastic energy terms.

\section{References}

[1] A. Planes, T. Castan, J. Ortin, L. Delay, J Appl Phys 66(6), 2342 (1989)

[2] K. Tanaka, Res. Mech. 18, 251 (1986)

[3] J. Van Humbeck and R. Stalmans, in K. Otsuka and C. M. Wayman (editors): Shape memory materials, Cambridge, Cambridge University Press, 1998, p. 151,

[4] S. Leclercq and C. Lexcellent, J.Mech.Phys.Solids, 44(6), 953 (1996):

[5] C. Lexcellent, M.L. Boubakar, Ch. Bouvet, S. Calloch, Int. J. of Sol. and Struct. 43, 613 (2006)

[6] D.L. Beke, L. Daróczi, Z. Palánki, Proc. of Int. Conf. on Shape Memory and Superleastic Technologies, 2007

Tsukuba, Japan, edited by S. Miyazaki (ASM International, Materials Park, Ohio, 2008) p. 607

[7] Z. Palánki, L. Daróczi, C. Lexcellent, D.L. Beke, Acta Mater 55, 1823 (2007)

[8] Z. Palánki, L. Daróczi, D.L. Beke, Mater. Trans. A46, 978 (2005)

[9] L. Daróczi, Z. Palánki, S. Szabó, D. L. Beke, Material Sci. and Eng. A378, 274 (2004)

[10] J. Rodriguez-Aseguinoza, I. Ruiz-Larrea, M.L. No, A. Lopez-Echarri, J. San Juan, Acta Mater 56, 6283 (2008)

[11] T.Y. El Rasasi, L. Daróczi, D.L. Beke, Material Sci. and Eng. A, submitted for publication

[12] L. Delaey, Diffusionless transformations” in R.W Cahn, P.Haasen and E.J. Kramer (editors) „, Materials Science and Technology - A Comprehensive Treatment" Vol. 5. P. Haasen (ed.) Phase Transformations in Materials, (Weinheim, VCH, 1991) p. 339.

[13] Tong HC, Wayman CM. Acta Metall 22, 887 (1974).

[14] Salzbrenner RJ, Cohen M. Acta Metall 27, 739 (1979)

[15] Chernenko, V. A., L'vov, V. A., Phil. Mag. A, 73, 999 (1996)

[16] Johari, G. P., McAnananma, J. G., Sartor, G., Phil. Mag. B, 74, 243 (1996) 